\title{
L'autre dans le discours politique, une stratégie de valorisation vs dévalorisation
}

\author{
Françoise Favart ${ }^{1} *$ \\ ${ }^{1}$ Iuslit, Università degli Studi di Trieste, 34127 Trieste, Italie
}

\begin{abstract}
Résumé. Il n'est pas rare que les acteurs politiques aient recours à des stratégies discursives dans lesquelles ils «disent» l'autre. À travers l'analyse d'un corpus constitué d'articles tirés du blog personnel de J.-L. Mélenchon nous montrerons comment les discours d'autrui, qui peuvent renvoyer à des figures de différentes natures (personnages historiques ayant marqués l'histoire ou acteurs politiques contemporains) constituent des stratégies argumentatives de valorisation $v s$ dévalorisation.
\end{abstract}

\begin{abstract}
Tell the other, a valuation strategy $v$ s devaluation. It is not uncommon for political actors to resort to discursive strategies in wich they "say " the other. Through the analysis of a corpus composed of articles taken from J.-L.Mélenchon's personal blog, we will show how the other's discourse, which can be presented under different figures (historic characters who have marked history or contemporary political actors) can constitute strategies of valuation vs devaluation.
\end{abstract}

\section{Introduction}

Le recours au discours d'autrui visant à favoriser l'adhésion à des propos énoncés n'est certes pas un procédé discursif nouveau (Perelman et Olbrechts-Tyteca 1988: 5). Récemment, lors de la campagne présidentielle de 2017, nous avons observé que les citations ou l'évocation de personnages illustres avaient fréquemment été utilisées par les candidats. Nous pensons entre autres à J. Jaurès cité par E. Macron à l'occasion du discours qu'il prononça à Albi ou à une allocution de J.-L. Mélenchon de mars 2017 où il évoquait, place de la République, à Paris, l'anniversaire du soulèvement de la Commune en citant longuement V. Hugo. Nous savons par ailleurs qu'il existe un lien entre le recours au discours d'autrui et la valorisation de soi dans certaines formes de discours politique telles que le débat parlementaire (Van Dijk et Wodak 2000: 66). Il n'est en outre plus à démontrer que cette valorisation passe aussi par la dévalorisation de l'autre comme l'a souligné C. Kerbrat-Orecchioni, notamment pour les débats de l'entre-deux-tours des élections présidentielles (2017 : 317). Partant de ces postulats, nous montrerons que de telles stratégies trouvent également leur place dans les blogs de politicien, une forme particulière de dispositif de communication politique où les instances médiatique et politique se rejoignent. Une caractéristique qui, dans la construction de l'ethos ${ }^{1} \mathrm{du}$ politicien, pourrait favoriser l'émergence d'une parole individuelle et personnalisée au détriment de la parole collective et limiter la présence de l'autre.

Notre réflexion s'appuiera sur le blog personnel du chef de file de La France insoumise dont nous analyserons une dizaine de " notes de blogs », allant de février 2017 à novembre 2019. Nous proposerons ainsi une analyse qui reposera sur des paroles d'autrui présentées

\footnotetext{
*ffavart@units.it
} 
sous la forme de discours rapporté, de type direct, qui peut être considéré comme un fait dialogique prototypique dans la mesure où il tend à expliciter l'interaction entre l'énonciation enchâssante et l'énonciation enchâssée (Bres et al 2019: 179). Nous tiendrons également compte du discours évoqué. Nous précisons toutefois que, le questionnement qui anime notre étude étant axé sur une visée argumentative, à savoir comment la parole des autres peut constituer un élément de valorisation $v s$ dévalorisation, nous n'étudierons pas le discours d'autrui dans ses différents aspects formels mais plutôt en tant que formulation du discours au service de l'argumentation (Sullet-Nylander et Roitman 2011 : 126). Dans tous les cas, qu'il s'agisse de citation directe ou d'évocation de propos d'autrui, nous nous limiterons à étudier les énoncés où l'autre est désigné de manière explicite, dans la majorité des cas, par un nom propre. De fait, s'il apparaît que la référence à autrui s'accompagne inévitablement d'une finalité argumentative, celle-ci concerne non seulement le choix des paroles rapportées mais peut-être plus encore le choix de l'autre que l'on cite. Nous déclinons ainsi les discours d'autrui en deux catégories majeures. La première est constituée d'autres qui s'apparentent à des figures historiques qui constituent un univers commun de références, en partage entre l'auteur du blog et ses lecteurs. La seconde renvoie à des acteurs politiques contemporains auxquels, dans le plupart des cas, s'oppose J.-L. Mélenchon.

Après un bref positionnement théorique dans lequel nous tenterons de circonscrire ce que nous entendons par blog de politicien et par discours d'autrui, nous préciserons les caractéristiques du corpus sur lequel repose notre analyse. Dans la partie finale de cet article, nous montrerons comment s'opèrent les stratégies de valorisation $v s$ dévalorisation et en quoi elles sont étroitement liées aux deux catégories de discours d'autrui précédemment évoquées.

\section{Positionnement théorique}

\subsection{Le blog du politicien dans l'espace de circulation du discours}

À l'heure où les acteurs du monde politique manifestent la volonté d'opérer une communication de plus en plus directe avec l'instance citoyenne, le blog semble constituer un support médiatique privilégié.

Au plan formel, ce nouveau canal communicationnel peut être défini comme un carnet de bord en ligne proposant un point de vue, qui peut être individuel ou collectif, sur la vie de la Cité, en réaction à des événements (Greffet 2005 : 5). Ce genre émergent se présente en outre sous différentes formes parmi lesquelles on distingue le blog personnel du blog d'affaires publiques (Miller et Shepherd 2009). Le premier se rattachant à l'exposition de la vie privée en public et au désir de construire une image de soi, alors que le but du second est l'action et le changement social. Deux catégories qui, dans le blog de politicien se croisent et se rejoignent parfois. En effet, au-delà d'informer le lecteur sur ses prises de position et ses activités en tant que personnalité élue, l'auteur de ce type de carnet de bord adopte également des attitudes militantes en faveur ou contre certaines réalités sociales ou politiques.

$\mathrm{Au}$ plan discursif, le blog constitue une instance de médiation qui participe de la circulation de la parole politique dans la mesure où il met en présence une instance politique et une instance citoyenne (Charaudeau 2014 : 42) et concourt à la construction de l'image de soi. Il peut être envisagé comme un genre de discours au sens où il est le reflet d'une pratique sociale ou un prototype de situation communicative récurrente (Miller 1984). M. Bonhomme considère pour sa part que dans le cas d'Internet, la généricité est ambivalente. En effet, d'une part elle se nourrit des genres en vigueur, mais de l'autre elle remodèle profondément les genres dont elle s'inspire, les enrichissant par de nouveaux 
invariants qui les transforment en genres numériques (2015:44). Nous ajouterons encore que cette forme de circulation du discours politique implique un positionnement tant dans la sphère politique que médiatique qui repose sur une auto-médiation. Il s'agit là, nous semble-t-il d'un trait caractérisant du blog de politicien qui ne relève pas tant du double positionnement, fréquent dans le discours politique, mais de l'auto-médiation où le politicien, auteur du blog est le maître du jeu à deux niveaux. De fait, dans le dispositif de la communication politique, il s'identifie conjointement à l'instance politique et à l'instance médiatique en évitant le filtre que constituent les médias traditionnels.

\subsection{L'autre que l'on cite ou que l'on évoque}

Il est plutôt malaisé de définir l'autre tant le terme renvoie à une multitude de sens et à des domaines aussi vastes que variés. Toutefois, si nous inscrivons notre réflexion dans le champ de l'analyse du discours telle qu'elle s'est développée en France, nous envisageons l'autre en tant que discours d'autrui que nous mettons en relation à deux concepts majeurs. Le premier s'appuie sur la distinction entre discours externe et discours interne (Bres 1999: 71) ou entre les dimensions dialogale et dialogique du discours telles que les a définies M. Bakhtine (1934). Le dialogue externe est marqué par l'alternance des tours de parole d'énonciateurs différents, le dialogue interne ou dialogisme est quant à lui à entendre comme « cette dimension constitutive qui tient à ce que le discours ne peut pas ne pas se réaliser dans un dialogue implicite avec d'autres discours » (Bres 1999 : 71).

La notion de dialogisme ou de présence du discours d'autres est également prise en compte par D. Maingueneau dans ce qu'il appelle interdiscours. L'auteur souligne le fait que «tout discours est traversé par l'interdiscursivité, il a pour propriété constitutive d'être en relation multiforme avec d'autres discours » (2002:324). Ainsi, tout discours, quel qu'il soit, ne saurait être traité dans le cadre de ses seules clôtures internes (Détrie et al. 2017 : 186-187). Il apparaît par ailleurs que la relation entre le discours et l'interdiscours s'articule sous des modalités différentes et que cette relation peut être d'ordre implicite ou explicite. En outre, s'il est évident que sa constitution est discursive, elle est également idéologique (Détrie et al. 2017 : 187) ce qui laisse entendre que la présence de l'interdiscours ne peut être séparée de la notion de point de vue et du positionnement adopté par l'énonciateur. Comme le soulignait déjà Bakhtine, les mots d'autrui, introduits dans notre discours, s'accompagnent immanquablement de notre attitude propre et de notre jugement de valeur (1970 : 269). On voit bien dès lors comment ces mécanismes de dédoublement des voix peuvent relever de stratégies discursives. De fait, le recours à une parole antérieurement prononcée par un autre ou par soi-même est-il susceptible d'en produire la redéfinition et le retournement, mais aussi d'en provoquer éventuellement l'effacement, l'oubli ou même la dénégation (Maingueneau 1987: 83). Il en résulte ainsi que la présence du discours d'autrui dans un discours propre s'accompagne inévitablement d'une finalité argumentative de la part de l'énonciateur qui le cite. Celle-ci concerne non seulement le choix des paroles rapportées mais peut-être plus encore, comme nous l'avons signalé précédemment, le choix de l'autre qui est à l'origine des propos cités.

D'une part, l'autre peut constituer une figure historique en partage entre l'énonciateur qui cite le discours d'autrui et les destinataires auxquels il s'adresse. Il génère alors un rapprochement, une connivence entre l'un et l'autre. Cette figure d'autorité relève en général d'un passé littéraire ou historique glorieux et valorise celui qui le cite en lui conférant de la légitimité. D'autre part, le discours d'autrui, peut renvoyer à des personnalités contemporaines, qui s'identifient souvent, dans le cas du discours politique, à des adversaires. Comme nous l'avons rappelé dans l'introduction, diverses modalités existent pour reprendre la parole de l'autre ou simplement pour en signaler la présence dans le discours, nous nous y intéresserons ici en tant que support à une argumentation visant à la valorisation ou à la dévalorisation. 


\section{Précisions sur le corpus et analyse}

\subsection{Le corpus}

Le blog personnel des politiciens semble répondre à une nouvelle modalité de communication et de rapprochement avec l'instance citoyenne où l'homme politique endosse à la fois le rôle d'instance médiatique et d'instance politique. Celui de J.-L. Mélenchon dont l'interface a été repensée en 2015, rencontre en moyenne 370000 visites par mois. À la date du 17 mars 2020, il en indiquait 372806 , en référence au mois de février 2020. Le blog s'articule autour de 10 onglets renvoyant à des sections distinctes intitulées : vidéos, articles, à l'assemblée, etc. Dans ces différentes sections, le politicien réagit à des faits d'actualité pouvant aller de la politique nationale à l'économie européenne ou encore à des faits de société pour ne citer que quelques exemples. Pour constituer le corpus sur lequel repose notre analyse, nous nous sommes intéressée à la rubrique Articles et en particulier à la sous-section intitulée Mes notes de blog. Nous avons ainsi retenu onze documents qui reposent sur un critère chronologique (la période allant de février 2017 à novembre 2019) plus que sur des critères thématiques. Nous avons toutefois privilégié, sans exclure totalement la sphère internationale, les articles touchant la politique nationale et constituant des sujets qui étaient au cœur de l'actualité de cette période. Ce choix s'explique par la volonté d'analyser la présence du discours d'autrui dans des thèmes variés et sur une période de plus de deux années afin de vérifier si le mécanisme argumentatif peut être considéré comme une stratégie récurrente dans un dispositif discursif où instance politique et instance médiatique fusionnent. Le corpus nous a en outre permis de remarquer que le discours d'autrui fréquemment évoqué par J.-L. Mélenchon renvoyait à des profils distincts qui peuvent se répartir sur les deux catégories majeures évoquées plus haut. Pour la première, il s'agit de figures marquantes de la culture française ou internationale, des hommes de lettres tel V. Hugo ou des hommes politiques ayant compté pour l'histoire de la Nation tels Robespierre, Napoléon ou le Général de Gaulle. Bien que plus rare, un recours à des références historiques internationales, notamment à travers la figure de Lénine, a également été observé. Pour la seconde, nous avons relevé la présence d'acteurs du monde politique contemporain, essentiellement des représentants du gouvernement d'E. Macron ou de personnalités qui lui sont proches et qui s'apparentent, sinon à des adversaires politiques directs de J.-L. Mélenchon, pour le moins à des antagonistes. L'une et l'autre de ces catégories ont en commun de renvoyer à des références partagées par la plupart des Français et plus certainement encore par la majorité des lecteurs du blog de J.-L. Mélenchon. Nous avons par ailleurs observé que le recours au discours d'autrui, en particulier en rapport à des figures contemporaines, est une stratégie discursive qui permet à J.-L. Mélenchon de s'auto-citer.

\subsection{Analyse}

\subsubsection{L'autre en tant que figure historique}

(1) Pour Victor Hugo d'ailleurs, seul l'auteur était légitime à percevoir des droits. Il écrivait ainsi en 1878 "L'héritier du sang est l'héritier du sang. L'écrivain, en tant qu'écrivain, n'a qu'un héritier, c'est l'héritier de l'esprit, c'est l'esprit humain, c'est le domaine public. Voilà la vérité absolue. ». Pour ma part je ne me prononce pas pour la suppression des droits d'auteurs pour les héritiers car à cette étape je ne veux rien brusquer : il s'agit de faire avancer une idée dans le débat public. (Mélenchon, 2017)

Dans un débat de 2017, sur les droits d'auteur et après avoir évoqué l'institution d'un « domaine public commun », J.-L. Mélenchon, favorable à la réduction de la durée de la perception de ces droits pour les héritiers, évoque V. Hugo tant en le nommant qu'en le 
citant. Le recours à une telle figure historique constitue un argument d'autorité. En effet, en signalant que près d'un siècle et demi plus tôt, V. Hugo énonçait des propos proches des siens, J.-L. Mélenchon légitime sa position et opère une valorisation de soi en se donnant à voir comme un héritier de l'homme de lettres.

(2) La France, depuis le Général de Gaulle, se défend toute seule. Notre appartenance à "l'Alliance transatlantique » ne se justifie pas. Elle nous met dans le camp de ceux qui, comme Trump et madame Kramp-Karrenbauer, sont des semeurs de guerre par leur agressivité inutile contre la Russie. (Mélenchon, 2019e)

L'extrait du point (2) présente la réaction de J.-L. Mélenchon à une tribune publiée par Annegrett Kramp-Karrenbauer, présidente du parti allemand $C D U$. Selon l'auteur du blog, cette tribune sonne comme un rappel à l'ordre à l'égard de la France face à son engagement au plan européen. Le député de La France insoumise souligne que celle-ci avait été publiée le jour où l'Assemblée nationale discutait une proposition de résolution sur un accord parlementaire franco-allemand. C'est à travers le nom du Général de Gaulle et l'évocation implicite d'épisodes de l'histoire de France que J.-L. Mélenchon tente de rendre légitime une position hostile à l'appartenance à l'Alliance transatlantique et souligne un désaccord face à la collaboration entre l'Allemagne et la France, évoqué dans d'autres articles du blog. Comme à l'exemple précédent, il s'auto-valorise par le biais d'une filiation avec de Gaulle.

(3) C'est pourquoi nous avons appelé ces révolutions «citoyennes ». Ici l'adjectif définit à la fois le moyen et la finalité. Et c'est comme ça qu'on le comprenait dès 1791, avec le premier monsieur Drouet. (Mélenchon, 2019a)

(4) Comment est-ce possible ? Drouet ? Cet homme est déjà passé devant nous. Il porte le nom d'un personnage dont Napoléon a dit « sans vous l'histoire de France aurait été toute différente ». Car il y a déjà eu un Drouet décisif dans l'histoire révolutionnaire de la France. Drouet c'est cet homme qui a observé attentivement cette diligence bizarre sur la route de Varennes en juin 1791. Il a reconnu le passager. C'était le roi Louis XVI fuyant Paris, le peuple et la révolution. (Mélenchon, 2018c)

Les deux extraits ci-dessus ont en commun d'évoquer Jean-Baptiste Drouet, un personnage historique qui ne figure pas parmi les plus connus de la Révolution française mais dont le nom est repris par J.-L. Mélenchon en raison de son homonymie avec Éric Drouet, figure emblématique des gilets jaunes. Dans le premier extrait, le nom du révolutionnaire, précédé de l'appellatif monsieur est uniquement cité. Ce procédé permet toutefois à l'énonciateur des propos citant d'établir, notamment grâce à l'adjectif premier - qui implicitement indique qu'un autre Drouet existe - un parallélisme entre les deux figures. De fait, si dans le premier extrait, la posture du révolutionnaire est simplement mentionnée, dans le second, elle revêt une forme de glorification. C'est à travers une citation de Napoléon que J.-L. Mélenchon complète le portrait de J.-B. Drouet. Nous assistons, dans ce cas à une double légitimation, puisque d'une part J.-L. Mélenchon s'appuie sur la figure historique de J.-B. Drouet pour justifier une position favorable au mouvement des gilets jaunes, de l'autre il a recours à une citation valorisante de Napoléon qui voit en J.-B. Drouet un personnage déterminant pour l'histoire de France.

(5) Naturellement, ce revolver et toute la violence de la scène ont été peints pour déprécier le mouvement populaire et stigmatiser sa «violence ». Et ce tableau 
n'est d'ailleurs exposé que pour cela encore aujourd'hui. Pourtant, pour ma part chaque fois qu'un groupe de visiteurs passe par là et que je m'y trouve, je lui commente le tableau. Et je lui parle de ce Drouet comme d'un modèle de citoyen qui sait aller à l'action révolutionnaire sans tortiller, pour faire vivre la République. «Ce n'est pas légal » hurlaient les réactionnaires de l'époque. «Et alors ? » demande Robespierre. La chute de la Bastille non plus, réplique-t-il, la proclamation de la fin de la royauté pas davantage ! Vous vouliez une révolution sans révolution » interroge Robespierre ? (Mélenchon, 2018c)

C'est ce que semble confirmer l'extrait ci-dessus, tiré d'un article qui débute par : «La révolution citoyenne des gilets jaunes est une des meilleures choses qui nous soit arrivée depuis si longtemps » et qui se poursuit par l'évocation d'un tableau exposé à l'Assemblée nationale où figure J.-B. Drouet. L'extrait se termine par une citation de Robespierre. On voit bien à travers ce cheminement que le nom propre Drouet sert de mise en relation entre les deux mouvements de mécontentement populaire et que les propos de Robespierre, qui justifient la chute de la Bastille, fonctionnent comme un argument d'autorité qui vise à conforter et à valoriser la position défendue par J.-L. Mélenchon. On y remarque clairement une implication idéologique où se dessine un parallélisme entre la Révolution française qui a porté au renversement du pouvoir établi et le mouvement de gilets jaunes que J.-L. Mélenchon soutient et encourage. Ce même parallélisme s'observe entre Robespierre et le chef de file de La France Insoumise. C'est encore de révolution qu'il est question dans l'extrait ci-après.

(6) D'ici à samedi, le sort du gouvernement va se jouer dans le rythme auquel vont s'agglomérer des mobilisations de tous les types à mesure que les sujets de mécontentements et les enjeux corporatifs seront vivifiés par la puissance du mouvement des gilets jaunes, [...]. « En haut on ne peut plus, en bas on ne veut plus ». La formule de Lénine pour décrire les situations pré-révolutionnaires est mise en place. (Mélenchon, 2018a)

Ici encore, le recours au discours direct, à travers la citation de Lénine, résume une situation de crise qui souligne l'impossibilité ou l'incapacité de la part du gouvernement et/ou du chef de l'État de réagir de manière appropriée et de l'autre le mécontentement populaire. En citant Lénine, J.-L. Mélenchon se fond dans la peau du révolutionnaire russe et prend à son compte, les propos de ce dernier. Ce faisant, il s'investit dans la posture d'une figure historique d'autorité pour renforcer son image de guide et de chef à l'égard des personnes qui adhèrent aux idées de La France insoumise.

\subsubsection{L'autre en tant qu'acteur contemporain}

(7) Il faudrait non pas un mais quatre jours de travail gratuit pour que le compte y soit. La ministre des Solidarités et de la Santé a proposé une autre méthode, tout aussi injuste et anti-sociale. Lors de la remise du rapport, elle s'est adressé[e] aux Français pour leur dire qu'il «faudra travailler un peu plus ». (Mélenchon, 2019f)

Après avoir exposé les difficultés rencontrées par la ministre des Solidarités et de la Santé, A. Buzyn, pour trouver 10 milliards d'euros supplémentaires, J.-L. Mélenchon souligne l'inutilité de la mesure et fait part de la proposition qui consisterait en l'allongement de la période de travail. En insérant, sous la forme du discours direct, les propos de la ministre, l'auteur du bloc, renforce sa crédibilité à l'égard du destinataire de son message. De fait, la citation souligne qu'il n'invente rien et que ce sont bien là les propos d'A. Buzyn. Ces paroles rapportées jouent un double rôle où d'une part elles confirment la validité du 
jugement de J.-L. Mélenchon et de l'autre elles impliquent directement le destinataire en lui permettant de se rendre compte personnellement que les méthodes proposées sont injustes et antisociales.

(8) Dans l'armée, on ne se cacha plus le malaise devant des " consignes très précises » de ce type. Du coup, toute la Macronie a rétropédalé. Aussitôt, la ministre des Armées indique qu'il " n'est dans l'idée de personne de mettre les militaires en face des manifestants ». Et Macron de dénoncer « un faux débat ». Rien de moins. Démentant Griveaux et le gouverneur militaire, il précisa sans vergogne que l'armée ne serait « en aucun cas en charge du maintien de l'ordre et de l'ordre public ». Donc le gouverneur militaire a menti ? Non, bien sûr. (Mélenchon, 2019d)

L'extrait proposé ci-dessus est la continuité d'un long passage, que nous avons coupé pour des raisons d'espace, dans lequel il apparaît que B. Griveaux, porte-parole du gouvernement, avait annoncé que des militaires se joindraient aux forces de l'ordre lors des manifestations des gilets jaunes. Une affirmation à laquelle le gouverneur militaire aurait répondu que les consignes [étaient] extrêmement précises. C'est sur la précision des consignes que J.-L. Mélenchon construit son argumentation en rapportant à la fois les propos du ministre des Armées et ceux du Président de la République. D'une part il a recours au discours direct, de l'autre il fait allusion, à travers ce qui peut être considéré comme une forme de discours évoqué (Charaudeau 1992 : 622), aux propos de B. Griveaux et du gouverneur militaire. Il ressort de cet entrelacs de paroles d'autrui une impression de désaccord entre les acteurs évoqués. En jouant sur la mise en scène d'un désaveu de la parole dite et sur le discrédit jeté sur le porte-parole du gouvernement et sur le gouverneur militaire, J.-L. Mélenchon fait émerger une image dévalorisante des dirigeants qui apparaissent alors comme peu fiables.

(9) Jusqu'ici, les Verts n'étaient pas invités dans cette combine. Mais la tête de liste d'Europe Ecologie/ les Verts veut désormais être de la partie. Au journaliste qui lui demande «dans le futur Parlement européen, les Verts feront-ils partie de la majorité quadripartite avec le PPE, les libéraux et les sociauxdémocrates? ", il répond sans détours. "Si on nous propose un programme qui améliore substantiellement le fonctionnement de l'Union et les politiques européennes, alors, EELV y apporteront leur soutien ». On peine à y croire : trois mois avant les élections, il annonce que les élus EELV feront une alliance avec le parti de Merkel et Orban, le parti de Macron et la social-démocratie en déroute. C'est d'autant plus étonnant que, dans la même interview, il critique, à raison, les accords de libre-échange signés tous azimuts par l'Union européenne. (Mélenchon, 2019c)

C'est ici sur une forme d'incohérence dans les propos tenus par la tête de liste du parti Europe Écologie/les Verts que J.-L. Mélenchon fonde sa critique. D'une part, il rappelle les mots de Y. Jadot en le citant et en signalant qu'il prend part à une combine. De l'autre, pour montrer ou l'incohérence ou l'incapacité de jugement du candidat, il évoque à travers le syntagme verbal il critique, d'autres paroles prononcées par Y. Jadot à l'occasion de la même interview. Comme dans l'exemple précédent, il en ressort l'image d'un candidat peu fiable. On remarquera par ailleurs que les choix lexicaux présents au plan de l'énonciation citante tels que combine et en déroute participent eux aussi à la construction d'une image dévalorisante du candidat d'EELV. Celle-ci se reflète également sur les trois autres politiciens auxquels il est associé (Merkel, Orban et Macron). 
(10) Cette position étrange de la part des EELV traduit en fait une profonde erreur d'analyse [...]. Dans son interview pour l'hebdo «Le Point», il déclare «bien entendu, les écologistes sont pour le commerce, la libre-entreprise et l'innovation ». Il va à l'encontre de la prise de conscience générale du lien entre le modèle économique actuel et la destruction de notre écosystème. (Mélenchon, 2019c)

En rapportant, plus loin dans l'article, la déclaration de Y. Jadot qui confère de la crédibilité et de la clairvoyance à J.-L. Mélenchon, ce dernier met en avant une autre forme d'incohérence. Non plus entre les propos qu'il a prononcés mais entre sa position d'écologiste et une prise de conscience "générale " contre les modèles économiques libéraux. Le chef de file de La France insoumise accentue ce faisant le portrait négatif de Y. Jadot et valorise la position de celui qui est capable de dénoncer cette profonde erreur d'analyse.

(11) Il a annoncé une hausse de Smic de 100 euros « sans qu'il en coûte un euro de plus aux employeurs » ce qui est évidemment impossible. Et pour cause, c'est un mensonge. Le salaire minimum n'augmentera pas plus que le minimum prévu par la loi au $1^{\mathrm{er}}$ janvier 2019. [...]. Et si les employeurs ne sortiront pas « un euro de plus », c'est bien parce que ce sont les contribuables qui vont payer. (Mélenchon, 2018b)

Dans l'extrait ci-dessus, tiré d'un article intitulé Les comptes tordus de Macron, J.-L. Mélenchon se sert d'une formulation au discours direct pour démentir les propos du Président de la République. Tout d'abord en affirmant, de manière péremptoire, que ce que le chef de l'État propose n'est pas possible et en accusant ce dernier de proférer un mensonge. Il se sert ensuite d'une seconde citation pour insinuer la peur chez le contribuable en affirmant que la hausse du Smic sera financée par les citoyens. Ce mécanisme argumentatif dévalorise d'une part le Président de la République, en le rendant peu crédible; de l'autre il valorise l'image de J.-L. Mélenchon qui apparaît comme une personne informée sur les faits et qui dit les choses telles qu'elles sont. Il renforce ainsi son ethos de crédibilité ${ }^{2}$.

\subsubsection{L'autre pour se dire soi-même}

Nous avons remarqué que dans plusieurs articles publiés sur son blog, l'évocation d'acteurs politiques contemporains était un moyen pour J.-L. Mélenchon de s'auto-citer ou s'autoévoquer.

(12) Le fameux prétendu « modèle allemand " est entré dans la phase que connaissent toujours les vieilles chaussettes : les trous deviennent visibles. L'économie allemande est mal en point. [...]. Pour l'année 2019, les prévisions ne sont pas meilleures : un petit $0,7 \%$ selon l'OCDE [...]. Alors qu'on nous vantait son industrie indestructible et ses excédents infinis, il vacille. En fait, cette difficulté pour l'économie outre-Rhin était prévisible. Les causes sont présentes depuis longtemps. Je les ai exposées depuis des années et notamment en 2014 dans mon livre « Le Hareng de Bismarck». (Mélenchon, 2019e)

Le discours évoqué renvoie ici à une institution, l'OCDE mais aussi à un énonciateur qui derrière le pronom indéfini on n'est pas clairement spécifié. Le contexte politique du moment permet toutefois d'imaginer qu'il s'agit d'E. Macron ou de manière générale des 
partisans du «couple franco-allemand». Une union décriée à plusieurs reprises dans d'autres articles du blog du chef de file de La France insoumise. Les difficultés rencontrées par le système allemand sont l'occasion pour le politicien de rappeler qu'il avait anticipé les failles de ce prétendu modèle économique montrant ici encore à ses lecteurs sa capacité d'analyse et sa clairvoyance. Il se rend en outre d'autant plus crédible qu'il a développé le sujet dans un ouvrage dont il cite le titre.

(13) Le vendredi 22 mars, le gouverneur militaire de Paris, le général Bruno Leray a déclaré à propos du rôle des militaires: "Les consignes sont extrêmement précises. Ils ont différents moyens d'action pour faire face à toute menace. Ça peut aller jusqu'à l'ouverture du feu. [...] ». Des consignes "extrêmement précises ». De qui ? Données quand et dans quel cadre ? Personne ne le lui demandera. Le problème médiatique est plutôt « Mélenchon a-t-il exagéré une fois de plus ? ». C'est exactement ce dont avait besoin le pouvoir pour effacer ses traces encore fraîches sur le sentier de la guerre. (Mélenchon, 2019d)

En s'appuyant sur la citation du général Leray, le député de gauche saisit l'occasion pour rappeler qu'il est sans cesse actif pour relever les défaillances du gouvernement et souligner une modalité d'agissement qui consiste à attirer l'attention sur des propos excessifs de J.-L. Mélenchon plutôt que d'affronter un problème réel. Ce dernier met par ailleurs en avant son rôle de dérangeur. Il laisse ainsi entendre qu'à la différence de l'institution, lui dit les choses telles qu'elles sont. Par ce mécanisme, il met l'accent sur la véridicité de sa parole et renforce son ethos de crédibilité à l'égard des destinataires de son blog.

(14) Pour la cheffe de l'extrême-droite, "la remise en cause de nos institutions, y compris avec une prétendue $6^{\text {ème }}$ République me paraît totalement malvenue ». À ce moment, peu soucieuse de son coup de com' récent sur des soi-disant convergences, elle m'accusait d'être derrière ce mot d'ordre : «cette revendication fait écho de manière assez grossière au programme politique d'un candidat à la présidentielle ». (Mélenchon, 2019b)

M. Le Pen, désignée tout d'abord par sa fonction (la cheffe de l'extrême droite) ensuite par son nom, souligne, dans sa première citation, un désaccord face à une éventuelle $6^{\text {ème }}$ République. Parallèlement, l'actualité politique de l'époque signalait que la mise en place d'une éventuelle $6^{\text {ème }}$ République avait été évoquée par J.-L. Mélenchon. D’une part, la position de ce dernier est dévalorisée par la citation de M. Le Pen, qui considère la proposition comme malvenue et qui, dans la seconde citation, qualifie d'assez grossière la revendication des gilets jaunes qu'elle met en parallèle au programme politique du député de gauche. De l'autre, J.-L. Mélenchon ressort valorisé en rappelant à la fois sa position face à l'actuelle République et en soulignant qu'il a été candidat à la présidentielle. De plus cette valorisation de soi passe également par la dévalorisation de M. Le Pen qu'il qualifie de peu soucieuse, la rendant ainsi peu crédible aux yeux des lecteurs.

\section{Dire l'autre une stratégie de valorisation vs dévalorisation ?}

Si nous partons du postulat que le dialogisme est cette forme de dialogue implicite avec le discours d'autrui et que dans le corpus que nous avons analysé cette forme de discours peut intégrer différentes catégories que nous avons identifiées par deux dimensions majeures : l'autre en tant que figure institutionnelle de référence au plan historique et l'autre en tant 
qu'acteur contemporain de la vie politique, nous avons observé que citer l'autre, dans de plus rares cas l'évoquer, constituait une stratégie argumentative. De fait, citer des personnages historiques aussi célèbres que le Général de Gaulle ou V. Hugo constitue ce qu'il est courant d'appeler un argument d'autorité. Il s'agit en effet de références culturelles partagées qui se comportent, dans le discours, comme des éléments fédérateurs pour la majorité des Français, conférant ainsi de la légitimité au propos de J.-L. Mélenchon. Dans le cas de J.-B. Drouet, la référence ne peut être considérée spontanément comme partagée. Personnage historique certes, mais peu connu de la majorité des Français, son association au leader des gilets jaunes donne lieu de la part de J.-L. Mélenchon à la production d'un récit qui joue de l'homonymie pour tirer des analogies substantielles entre le passé et le présent. Le Président de La France insoumise instruit le destinataire moins féru d'histoire sur les valeurs républicaines que défendait ce personnage. Il se réfère ainsi à la Révolution française et à Robespierre qui, pour sa part, constitue bien une figure de référence. Si cela ne suffisait pas, J.-L. Mélenchon s'appuie également sur une citation de Napoléon $1^{\mathrm{er}}$, dans une tentative de justification des mouvements de mécontentements des gilets jaunes. Il a en outre recours à des propos prononcés par Lénine. À travers ces différents procédés, J.-L. Mélenchon opère une légitimation de son discours et par là même de sa position. De fait, il donne de lui l'image d'un homme instruit et prêt à se fondre dans la posture de ces hommes célèbres pour guider la France. Il contribue ainsi à se construire un ethos d'identification ${ }^{3}$ et en particulier un ethos de chef où on imagine qu'il se coulerait aisément dans la peau d'un révolutionnaire tel Robespierre, prêt à renverser le pouvoir établi.

En ce qui concerne, les citations des acteurs contemporains, nous avons remarqué qu'elles visaient essentiellement à une dévalorisation de leurs auteurs, en les rendant peu crédibles. Cette stratégie a notamment été observée à l'égard du chef de l'État, de certains de ses ministres de l'époque, comme A. Buzyn ou encore de l'alors porte-parole du gouvernement, B. Griveaux. Dans de nombreux cas, la stratégie de J.-L. Mélenchon repose sur la mise en évidence d'une incohérence soit parce que les propos cités font émerger des contradictions par rapport à des propos antérieurs, comme à l'exemple (8), soit parce qu'une citation, comme celle de la ministre des Solidarités et de la Santé ou celle d'Y. Jadot, est en inadéquation avec une situation globalement admise au plan national voire international. L'incohérence peut en outre être soulignée par rapport à une forme de bon sens commun, à ce qui apparaît comme une évidence aux yeux de tous (exemple 11). En utilisant de tels procédés, J.-L. Mélenchon place les auteurs des propos cités dans une position de porte-à-faux, allant parfois jusqu'à les accuser de mentir. En revanche, quand les citations renvoient à des politiciens étrangers du monde contemporain, ce n'est pas tant sur l'auteur des propos que porte la dévalorisation mais plutôt sur les adversaires directs du politicien de gauche. Ainsi, en renvoyant à des instances politiques allemandes et en évoquant les relations tant décriées entre la France et l'Allemagne ${ }^{4}$, J.-L. Mélenchon se sert de la citation d'un tiers pour toucher un adversaire politique français. On voit bien dès lors comment des propos cités participent d'une stratégie argumentative de dévalorisation de l'autre ou d'un adversaire politique par le biais d'un discours d'autrui.

Ailleurs dans notre analyse, nous avons observé que citer des figures contemporaines comme M. Le Pen, le général Leray ou encore une institution économique internationale telle l'O.C.D.E. permettait à J.-L. Mélenchon de s'auto-citer ou de s'auto-évoquer. Une stratégie par laquelle il montre aux lecteurs de son blog que sa présence est significative au sein de la scène politique. Il opère en outre cette valorisation de soi en soulignant sa clairvoyance et en faisant remarquer qu'il alimente le débat médiatique. De plus, il s'inscrit comme une figure active au plan médiatique et politique où il se montre favorable à un renouvellement notamment à travers la création de la $6{ }^{\mathrm{e} m e}$ République. Un positionnement qui se rapproche ici aussi de l'ethos de chef que nous avons mis en évidence pour les discours d'autrui se référant à des figures historiques. 


\section{Conclusion}

Pour conclure, il nous semble pouvoir affirmer qu'avoir recours au discours d'autrui participe bien d'une stratégie argumentative de valorisation de soi vs de dévalorisation de l'autre, au sens d'adversaire politique, et ceci même dans dispositif de communication qui aurait pu favoriser, dans la construction de l'ethos, l'émergence d'une parole individuelle au détriment de la parole d'autrui. Nous avons en effet observé que citer une figure de renom, constituant une référence partagée pour la majorité des Français permettait à J.-L. Mélenchon de valoriser son image, comme si, en citant un personnage illustre, l'énonciateur de l'énonciation citante ${ }^{5}$ revêtait à son tour les qualités de l'énonciateur cité. Toutefois, il est significatif de relever que dans le cas de figures illustres, l'effet de valorisation est pleinement dépendant de la référence choisie. Le cas de V. Hugo ou de Robespierre est en ce sens assez significatif. De fait, le processus de valorisation se situe davantage dans la connivence symbolique que le destinataire du message est censé relever entre «l'emprunteur » et «l'emprunté » que dans le discours lui-même. On pourrait même y voir un jeu plus complexe où l'usage et le mésusage de l'Histoire est devenu un enjeu politique qui vise à insérer le présent « dans les pas de l'Histoire » (Offenstadt 2014).

Par ailleurs, le mécanisme de reprise de la parole d'autrui constitue également une stratégie de valorisation dès lors qu'elle permet à l'énonciateur de premier niveau de revenir sur ses propos ou sur ses actes. En revanche, les citations attribuées à des personnalités politiques contemporaines visent essentiellement à en dévaloriser leurs auteurs, sauf quand elles constituent pour l'énonciateur de premier niveau un moyen pour s'auto-citer. Il en va globalement de même quand l'auteur de la citation ou des propos évoqués occupe le rôle d'un tiers qui n'a d'autre fonction que de dévaloriser la figure politique à laquelle ce tiers est associé. Un procédé qui, de manière plus vaste, relève de la joute politique et qui acquiert tout son sens dès lors qu'on le replace dans le contexte sociopolitique du moment.

Ainsi, dans l'argumentation la valorisation et la dévalorisation se rejoignent lors de la construction de l'image de soi que veut donner l'énonciateur de premier niveau. De fait, comme le rappelle R. Amossy "l'efficacité de la parole est liée à l'autorité de l'orateur » (1999: 127) ou du scripteur. Plus encore dans le cas d'un blog de politicien où le partage des idées entre l'émetteur et la majorité des destinataires relève d'une complicité forte.

\section{Références bibliographiques}

Amossy, R. (1999). Images de soi dans le discours. La construction de l'ethos. Lausanne-Paris : Delachaux Niestlé.

Bakhtine, M. (1934). Esthétique et théorie du roman. Paris : Gallimard.

Bakhtine, M. (1970). La Poétique de Dostoïevski. Paris : Points Essai.

Bonhomme, M. (2015). La problématique des genres de discours dans la communication sur internet. Travaux neuchâtelois de linguistique, $\mathrm{n}^{\circ} 63,31-47$.

Bres, J. (1999). Vous les entendez ? Analyse du discours et dialogisme. Modèles linguistiques, Association Modèles, tome $\mathrm{n}^{\circ} \mathrm{XX}$, fasc. 2, 71-86.

Bres, J., Nowakowska, A. et Sarale, J.-M. (2019). Petite grammaire alphabétique du dialogisme. Paris : Classiques Garnier.

Charaudeau, P. (1992). Grammaire du sens et de l'expression. Paris : Hachette éducation.

Charaudeau, P. (2014). Le discours politique. Les masques du pouvoir. Limoges : Lambert-Lucas.

Constantin de Chanay, H. (2010). La polyphonie au service de l'éthos. L'exemple du débat du 2 mai 2007 entre N. Sarkozy et S. Royal, Recherches en linguistique, n 31, 299-320.

Détrie, C., Siblot, P., Verine, B. et Steuckart, A. (2017). Termes et concepts pour l'analyse du discours. Une approche praxématique, Nouvelle édition augmentée. Paris : Honoré Champion.

Favart, F. (2019). La répétition et la construction de l'ethos dans le discours politique, in Druetta, R. et Paissa, P. (éds.). La répétition en discours. Louvain-La Neuve : Academia- L'Harmattan, 199- 
216.

Greffet, F. (2005). Politics as usual. Les blogs politiques français en 2005. Démocratie et dispositifs électroniques : regards sur la décision, la délibération et le militantisme, Actes du colloque international http://loiseaugerard.free.fr/DELcolloque/DEL,\%20militantisme/DEL\%2012,\%20Greffet, \%20blogs.pdf, (consulté le 5 mars 2020).

Kerbrat-Orecchioni, C. (2017). Les débats de l'entre-deux-tours des élections présidentielles françaises. Constantes et évolution d'un genre. Paris : L'Harmattan.

Maingueneau, D. (1987). Nouvelles tendances en analyse du discours. Paris : Hachette.

Maingueneau, D. (2002). Problèmes d'ethos. Pratiques, n 113-114.

Miller, C. R. (1984). Genre as Social Action. Quarterly Journal of Speech, n 70, p.151-176.

Miller, C. R. et Shepherd, D. (2009). Questions for genre theory from the blogosphere, in Giltrow, J. et Dieter S. (éds.). Genres in the Internet. Amsterdam : John Benjamins, 263-290.

Offenstadt, N. (2014). L'Histoire un combat au présen. Paris : Textuel.

Perelman, C. et Olbrechts-Tyteca, L. (1988) [1957]. Traité de l'argumentation: la nouvelle rhétorique. Bruxelles : Éditions de l'université de Bruxelles.

Van Dijk, T. et Wolak, R. (2000). Racism at the Top. Parliamentary Discourses on Ethnic Issues in Six European Countries. Klagenfurt: Drava Verlag.

\section{Corpus}

Melenchon, Jean-Luc, 2017, «Culture, je recycle une idée de Victor Hugo », https://melenchon.fr/2017/02/22/culture-je-recycle-une-idee-de-victor-hugo/, (consulté le 2 avril 2019).

- 2018a, «La dernière cartouche », https://melenchon.fr/2018/12/05/la-derniere-cartouche/, (consulté le 2 avril 2019).

- 2018b, «Les comptes tordus de Macron », https://melenchon.fr/2018/12/12/les-comptes-tordusde-macron/, (consulté le 2 avril 2019).

- 2018c, «Merci Monsieur Drouet », https://melenchon.fr/2018/12/31/merci-monsieur-drouet/, (consulté le 2 avril 2019).

2019a, «Drouet et le nouvel âge citoyen des révolutions », https://melenchon.fr/2019/01/04/drouet-et-le-nouvel-age-citoyen-des-revolutions/, (consulté le 2 avril 2019).

— 2019b, « Le Pen de six à cinq », (https://melenchon.fr/2019/01/28/le-pen-de-six-a-cinq/), (consulté le 2 avril 2019).

- 2019c, «Jadot ne s'en cache pas: retour à l'écologie de marché », https://melenchon.fr/2019/03/05/jadot-ne-sen-cache-pas-retour-a-lecologie-de-marche/, (consulté le 2 avril 2019).

— 2019d, «L'État humilié par la macronie », https://melenchon.fr/2019/03/26/letat-humilie-par-lamacronie/, (consulté le 2 avril 2019).

- 2019e, «La chaussette trouée allemande», https://melenchon.fr/2019/03/26/la-chaussette-troueeallemande/, (consulté le 3 avril 2019).

- 2019f, «La semaine piteuse de la macronie », (https://melenchon.fr/2019/04/01/la-semainepiteuse-de-la-macronie/), (consulté le 2 avril 2019).

\footnotetext{
${ }^{1}$ La notion d'ethos dans le discours politique ayant déjà été largement étudiée ailleurs, nous n'y revenons pas ici et renvoyons entre autres à D. Maingueneau (2002: 65), H. Constantin de Chanay, (2010), P. Charaudeau (2014: 88) et F. Favart (2019: 202).

${ }^{2}$ Nous nous appuyons sur les distinctions proposées par P. Charaudeau (2014:87-128) qui relève 3 types d'ethos de crédibilité : l'ethos de vertu, de sérieux et de compétence et 6 types d'ethos d'identification : l'ethos de puissance, de caractère, d'intelligence, d'humanité, de chef et de solidarité.

${ }^{3}$ Nous nous référons en particulier à l'ethos d'intelligence (Charaudeau 2014 : 112-114).

${ }^{4}$ Nous signalons à titre d'exemple l'article intitulé Traité stupide :

https://melenchon.fr/2019/03/12/sortez-des-traites-stupides-tribune-de-jean-luc-melenchon-enreponse-a-emmanuel-macron/ ou encore Le drôle d'ami allemand fait la loi : https://melenchon.fr/2019/03/05/cetait-cense-etre-des-vacances/, (consultés le 23 décembre 2019).
} 
${ }^{5}$ Plus loin, énonciateur de premier niveau en opposition à l'énonciateur de l'énonciation citée, considéré comme un énonciateur de second niveau. 